\title{
UM MODELO MACROSCÓPICO PARA A FORÇA DE REAÇÃO RADIATIVA
}

\author{
MÁRIO GOTO 1
}

GOTO, M. Um Modelo Macroscópico para a Força de Reação Radiativa. Semina: Ci. Exatas/Tecnológicas, Londrina, v. 16, n. 4, p. 563-568, dez. 1995.

RESUMO: Apresentamos um sistema macroscópico simples, consistindo de uma casca esférica com distribuição uniforme de cargas, no interior da qual é colocada uma carga teste. Mostra-se que a distribuição de cargas da casca esférica, quando acelerada, cria um campo eletromagnético não nulo que atua sobre a carga teste induzindo, em regiões próximas ao centro da casca esférica, uma força do tipo da reação radiativa de Lorentz-Dirac.

PALA VRAS-CHAVES: Força de reação radiativa; Força de Lorentz-Dirac; Dinâmica das partículas carregadas,

\section{Introdução}

0 eletromagnetismo é uma das teorias mais bem sucedidas da Física, tendo servido inclusive como modelo para a construção de outras teorias, e um dos resultados de maior alcance reside na previsão das ondas eletromagnéticas e a origem das mesmas, as cargas aceleradas [JACKSON, 1962; MARION, 1965].

No entanto, justamente a radiação eletromagnética e o seu efeito na dinâmica das partículas carregadas são os focos de problemas não totalmente resolvidos da eletrodinãmica [JACKSON, 1962; TEITELBOIM \& al, 1980], tais como a origem da força de reação radiativa $e$ a interpretação física adequada das conseqüências desta força, aprofundando-se na compatibilidade da presença desta força com o Princípio da Equivalência [ROHRLICH, 1963]. O Princípio da Equivalência, como sabemos, e o postulado básico da Relatividade Geral [WEINBERG, 1972], e afirma que todos os corpos, independentemente de sua natureza, sofrem a mesma aceleração gravitacionai, de modo que a questão surge ao considerarmos que a força de reação radiativa que atua sobre uma partícula carregada acelerada atua justamente no sentido de opor-se a esta aceleração, o que reduziria a aceleração gravitacionai das partículas carregadas [FULTON \& ROHRLICH, 1960].

A importância destas questões tem sido despertada mais recentemente ao relacioná-las com novos fenômenos, tais como o efeito Unruh [UNRUH, 1976] e a prevista radiação térmica detectada por observadores acelerados [UNRUH \& WALD, 1984; BARUT \& DOWLING, 1990], além da introdução da quantização nos referenciais acelerados [BIRRELL \& DAVIES, 1982].
A motivação deste trabalho é apresentar um modelo macroscópico simples que dê origem a uma força do tipo Lorentz-Dirac, e que pode servir de apoio para um enfoque novo na questão sobre a origem da força de reação radiativa de Lorentz-Dirac, e que pode representar uma importante mudança na visão filosófica de alguns aspectos da Física.

O modelo proposto consiste de uma partícula carregada (carga teste, com carga elétrica e) colocada no interior de uma casca esférica rígida não condutora com distribuição uniforme de cargas (com carga total -e, negativa em relação a carga teste). Em tal sistema, podese mostrar que o campo eletromagnético resultante no interior da esfera, devido a distribuição de cargas na casca esférica, é dado por uma combinação dos campos retardado e avançado de Lienard-Wiechert, não nulo apenas no caso da casca esférica estar acelerada. Neste caso, a carga teste no interior da casca esférica sentirá o efeito deste campo, e em região próxima ao centro da casca esférica, a força resultante e justamente do tipo da força de reação radiativa de Lorentz-Dirac.

Um sistema deste tipo sugere uma alternativa interessante para a interpretação da origem da força de reação radiativa de uma carga acelerada, algo como um efeito Mach eletromagnético. Deixamos tal possibilidade, que depende de uma compreensão mais aprofundada das propriedades de transformação das leis da Física, e em especial do eletromagnetismo, entre referenciais não inerciais (referenciais acelerados), para um estudo subseqüente, concentrando-nos neste trabalho na exploração do modelo proposto, com o objetivo de derivar a força do tipo Lorentz-Dirac a que está sujeita a carga teste.

1 - Departamento de Física/CCE - Universidade Estadual de Londrina, PR, Brasil, Caixa Postal 6001, CEP 86051-990

Semina Ci, ExatasíTecn., v. 16, rt 4, p. 563-568 
Tal derivação pode ser feita considerando apenas a força de Lorentz aplicada sobre o campo no interior da casca esférica, na situação onde momentaneamente a partícula carregada encontra-se próxima ao centro da esfera, com velocidade próxima a desenvolvida pela casca esférica.

Será adotada a sinalização $(-,+,+,+)$ para o tensor métrico $\mathrm{g}^{\mu \mathrm{v}}$.

\section{A força de reação radiativa}

É bem conhecido o fato de as partículas carregadas (carga e) emitirem radiação eletromagnética quando aceleradas, a potência total irradiada dada pela conhecida fórmula (não relativística) de Larmor [JACKSON, 1962]

$$
R(t)=\frac{2 \mathrm{e}^{2}}{3 \mathrm{c}^{3}} \mid v y^{2}
$$

ou pela sua generalização relativística de Lorentz

$$
R(\tau)=-\frac{2 e^{2}}{3 m^{2} c^{3}} \frac{d p_{-i}}{d \tau} \frac{d p^{\mu}}{d \tau}
$$

Em termos da energia-momento carregada pela radiação, temos

$$
\frac{d p_{r a d}^{\mu}}{d t}=\frac{2}{3} \frac{e^{2}}{c^{5}} a_{\lambda} a^{\lambda} v^{\mu}
$$

a fórmula de Larmor resultando

$$
R=-v_{\mu} \frac{d p_{r a d}^{\mu}}{d \tau}=\frac{2}{3} \frac{e^{2}}{c^{3}} a_{\lambda} a^{\lambda}
$$

que é essencialmente não nulo, exceto para aceleração nula.

A perda de energia-momento pela partícula carregada devido a emissão da radiação manifesta-se em forma da força de reação radiativa, obtida por considerações de conservação da energia e momento da partícula, assumindo, no caso não relatívístico, a forma

$$
F_{\text {rac }}=\frac{2}{3} \frac{e^{2}}{c^{3}} v=m \tau,
$$

Lorentz,

$$
m(v-\tau v)=F_{e x t},
$$

onde $F_{\text {ext }}$ é uma força externa.

Esta equação nem sempre leva a soluções fisicamente aceitáveis. Considere, por exemplo, o caso de uma força externa constante, que pode ser devida a um campo gravitacional uniforme,

$$
\mathrm{F}_{\text {ext }}=\mathrm{mg} \text {. }
$$

Neste caso, a equação de movimento fica

$$
r-\tau r=g,
$$

que, sendo g uma constante, pode ser escrita como

$$
(a-g)-\tau \frac{d}{d t}(a-g)=0
$$

cuja solução é

$$
a(t)=g+\left(a_{0}-g\right) e^{k t}
$$

Esta solução prevê $a(t=0)=a_{\circ}$ arbitrário, que não corresponde a uma solução fisicamente aceitável, exceto para o caso $a_{0}=g$, quando $a=g$, constante, que implica na força de reação radiativa nula, $r=0$.

Este é um resultado importante, pois mostra que a força de reação radiativa não invalida o Princípio da Equivalência (todos os corpos em queda livre num campo gravitacional devem sofrer a mesma aceleração, independente da natureza dos corpos envolvidos), pelo menos para o caso de um campo gravitacional uniforme. Este resultado é válido também na versão relativística, onde pode-se mostrar que a força de reação radiativa se anula no caso de cargas em movimento hiperbólico, resultante da ação de uma força constante [ROHRLICH, 1961]. Se $a_{\circ}$ g, a aceleração cresce exponencialmente com o tempo (solução auto-acelerada), não sendo portanto uma solução fisicamente aceitável. No caso $\mathrm{g}=0$, assume a conhecida forma

$$
V(t)=0 \quad\left\{\begin{array}{l}
0 \\
a_{0} e^{t / t}
\end{array}\right.
$$

Este é um exemplo típico das limitações da equa- 
ção de Abrahan-Lorentz. A solução fisicamente plausível seria uma desaceleração exponencial, que no entanto não é solução da equação diferencial [DIRAC, 1938].

Uma das formas propostas para se contornar esta dificuldade e transformar a equação diferencial \{??) numa equação integro-diferencial,

$$
m v(t)=\int_{0}^{\infty} e^{-s} F(t+\tau s) d s
$$

que, embora evite a auto-aceleração, traz uma outra dificuldade, subvertendo a causalidade, através de uma pré-aceleração, isto é, a resposta a força antes que a mesma tenha sido aplicada, embora argumente-se que o intervalo de tempo envolvido nesta quebra de causalidade esteja fora de alcance das medições experimentais da física clássica [JACKSON, 1962).

A versão relativistica da equação (6) pode ser obtida de várias maneiras, assumindo a forma [TEITELBOIM \& ai, 1980; LANDAU \& LIFSHITZ, 1980]

$$
\frac{d p^{\mu}}{d \tau}=f_{e x t}+\frac{2}{3} \frac{e^{2}}{c^{3}}\left[\frac{d^{2} U^{\mu}}{d \tau^{2}}-U^{\mu} U^{\mu} \frac{d^{2} U_{\gamma}}{d \tau^{2}}\right]
$$

\section{Sistema com força do tipo Lorentz-Dirac}

Vamos analisar aqui um sistema cuja interação resulta numa força do tipo Lorentz-Dirac, sem no entanto estar diretamente relacionada com emissão de radiação eletromagnética, que é a origem usual da força de reação radiativa.

O sistema proposto consiste de uma partícula com carga elétrica $q=e$ (carga teste) no interior de uma casca esférica rígida não condutora uniformemente carregada com carga total $Q=-e$. O conceito de corpo rígido, no contexto relativístico, deve ser empregado com um significado apenas aproximado, suficiente para os propósitos deste trabalho, onde o essencial e que, quando em repouso, a casca esférica e a distribuição de cargas sobre a mesma mantenha a simetria esférica, e quando em movimento, não sofra deformações que não as devidas a geometria do espaço co-tempo.

Em trabalho recente [GOTO, 1994], mostramos que uma casca esférica deste tipo, quando acelerado, contém em seu interior um campo eletromagnético não nulo, de modo que a carga teste irá interagir com este campo através da força de Lorentz,

$$
\frac{q^{\mu}}{c}=F^{\mu v} x_{v},
$$

o campo interno sendo uma combinação dos campos retardado e avançado,

$$
A^{\mu}=\frac{1}{2}\left(A_{\text {ret }}^{\mu}-A_{\text {adv }}^{\mu}\right)
$$

onde

e

$$
A_{r s t}^{\mu}=\frac{Q z^{\mu}}{P_{\text {ret }}}
$$

$$
A^{p}{ }_{\text {aov }}=-\frac{Q Z^{1}}{P_{\text {ad: }}}=
$$

para

$$
\rho=-\left(x^{\mu}-z^{\mu}\right) z_{\mu}=-R^{\mu} z_{\mu}
$$

Utiłizamos a notaçāo $z^{\mu}$ para as coordenadas do centro da casca esférica e $x^{\mu}$ para as coordenadas da carga teste. As derivadas

$$
\begin{aligned}
& \dot{x}^{\mu}(\tau)=\frac{d x^{\mu}}{d \tau} \\
& z^{\mu}(\tau)=\frac{d z^{\mu}}{d \eta}
\end{aligned}
$$

definem as respectivas quadri-velocidades; $\tau$ e $\eta$ sāo os tempos próprios da carga teste e da casca esférica, respectivamente.

Os campos retardados e avançados na posiçăo do espaço co-tempo $x^{\mu}$ devem ser calculados para

$$
\eta=\tau_{\text {rel }}=\tau_{*} \quad \text { e } \quad \eta=\tau_{\text {adv }}=\tau_{-} .
$$

respectivamente.

Em termos do tensor eletromagnético, temos

$$
F_{\mu v}=\frac{1}{2}\left(F^{*}{ }_{\mu v}-F_{\mu v}^{-}\right)
$$

onde

$$
F_{\mu \nu}^{ \pm}=\partial_{\mu \nu} A_{\nu}^{ \pm}=\partial_{v} A^{ \pm}{ }_{\mu}
$$

Para evitar uma superposição exagerada de índices, nas fórmulas válidas tanto para as grandezas retardadas ou para as avançadas, deixaremos de utilizar os índices ( \pm ) ou (ret/adv), desde que, evidentemente, 
tal procedimento não prejudique a clareza das expressões envolvidas.

Ao se efetuar as derivaçōes, devemos estar atentos ao fato de os tempos retardado e avançado, $\tau_{t}$, estarem relacionados com as coordenadas da posição onde os campos estāo sendo avaliados. Da rejaçāo

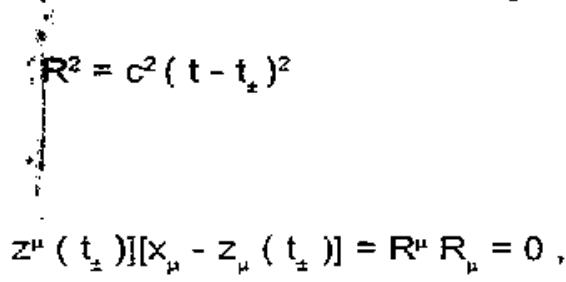

de onde resulta, por diferenciação,

$$
R^{\mu} d R_{\mu}=R^{\mu}\left[d x_{\mu}-z_{\mu} d \tau_{ \pm}\right]=0
$$

ou seja,

$$
d \tau_{ \pm}=\frac{\partial \tau_{ \pm}}{\partial x^{\mu}} \mathbf{d x}=\frac{R_{\mu}}{R^{\alpha} z_{\alpha}} d x^{\mu}
$$

Isto mostra que

$$
\frac{\partial \tau_{ \pm}}{\partial x^{\mu}}=\partial_{\mu} \tau_{ \pm}=-\frac{R_{\mu}}{P_{ \pm}}
$$

Esta fórmula deve ser considerada nas derivaçôes dos campos retardados e avançados, por exemplo,

$$
\partial_{\mu} A_{\nu}=\left(\partial_{\mu}-\frac{R_{\mu}}{P_{\underline{\mu}}} \frac{d}{d t_{ \pm}}\right) A_{\nu}
$$

onde $\partial_{\mu}$ indica a derivada em $x^{\mu}$ apenas onde a mesma apareça explicitamente na função.

Os campos retardados e avançados da equação podem ser representados por

$$
A_{ \pm}^{\mu}=\frac{Q z^{\mu}\left(\tau_{ \pm}\right)}{ \pm p_{ \pm}}
$$

resultando

$$
\partial_{\mu} A_{v}^{ \pm}=\frac{\mp Q^{2} Z U}{\rho^{2}} \partial_{\mu \rho} \pm \frac{Q^{2} Z U}{\rho} \partial_{\mu} \tau_{ \pm} .
$$

$$
z^{\mu} z_{\mu}=-c^{2} \Rightarrow \dot{z}^{\mu} z_{\mu}=0 \text { e } z^{\mu} z_{\mu}=-a^{2}
$$

derivação

$$
\partial_{\mu} \rho=-z_{\mu}+\frac{c^{2} R_{u}}{\rho}+\left(R^{\alpha} z_{\alpha}\right) \frac{R_{\mu}}{\rho}
$$

resulta

$\partial_{\mu} A_{v}^{ \pm}=\mp \frac{Q z_{v}}{\rho^{2}}-z_{\mu}+\frac{c^{2} R_{\mu}}{\rho}+\left(R^{\alpha} z_{\alpha}\right) \frac{R_{u}}{\rho} \mp \frac{Q z_{\nu} R_{\mu}}{\rho^{2}}$,

e portanto

$$
F_{\mu \nu}^{t}=\mp \frac{Q}{\rho^{3}}\left[c^{2}+(R a)\right]\left(R_{\mu} z_{\nu}-R_{\nu} z_{\mu}\right) \mp \frac{Q}{\rho^{2}}\left(R_{\mu} z_{\nu}-R_{\nu} z_{\mu}\right)
$$

lembrando que o campo resultante è a combinaçāo

$$
F_{\mu v}=\frac{1}{2}\left(F_{\mu v}^{+}-F_{\mu v}^{-}\right) \text {. }
$$

Se a carga teste estiver próxima ao centro da esfera, temos

$$
X^{\mu}(\tau) \equiv Z^{\mu}(\tau),
$$

sendo que interagirá com o campo retardado que no instante $\mathrm{t}$ - $\Delta$ t passou pelo centro da esfera e com 0 campo avançado que chegará ao centro da esfera no instante $t+\Delta t$, onde

$$
\Delta t=\frac{\left|r+r^{\prime}\right|}{c}=\frac{R}{c} \text {, }
$$

as coordenadas espaço co-temporais definidas por $\left(x^{\mu}\right)$ $=(\mathrm{ct}, \mathrm{r}) \in\left(\mathrm{z}^{\mu}\right)=\left(\mathrm{ct}^{*}, r^{\prime}\right)$, de modo que os campos retardado e avançado serão dados, respectivamente, por

$$
A^{\mu}{ }_{+}(X)=\frac{Q Z^{\mu}(\tau-\Delta \tau)}{\left[X^{\alpha}(\tau)-Z^{\alpha}(\tau-\Delta \tau)\right] Z_{a}(\tau-\Delta \tau)} .
$$

e

$$
A_{-}^{\mu}(x)=-\frac{Q Z^{\mu}(\tau+\Delta \tau)}{\left[x^{\alpha}(\tau)-Z^{\alpha}(\tau+\Delta \tau)\right] Z_{\alpha}(\tau+\Delta \tau)}
$$

A equação de movimento da carga teste fica

$$
m x^{\mu}=\frac{q}{c} F^{u v} k_{v},
$$




$$
z^{\mu}\left(\tau_{+}\right)=z^{\mu}(\tau-\Delta \tau)
$$

e o campo avançado na posição

$$
z^{\mu}(\tau)=z^{\mu}(\tau+\Delta \tau)
$$

No limite $\Delta \tau \rightarrow 0$, estabelecemos a dinâmica da carga teste próxino ao centro da casca esférica. Neste sentido, devemos fazer a expansão em potências de $\Delta \tau$ dos campos e calcular a força de Lorentz neste firnite [DIRAC, 1938].

Na medida do possível, usaremos notaçōes simplificadas do tipo

$$
v^{\nu}=z^{\mu}, a^{\mu}=z^{\mu},(r a)=r^{\mu} a_{\mu}, a^{2}=a^{\mu} a_{\mu}, \text { etc. }
$$

\section{Temos}

$Z^{\mu}(\tau \mp \Delta \tau)=z^{\mu}(\tau) \mp z^{\mu} \Delta \tau+\frac{1}{2} z^{\mu} \Delta \tau^{2} \mp \frac{1}{6} z^{\mu} \Delta \tau^{3}+\ldots$

e as derivadas temporais sucessivas

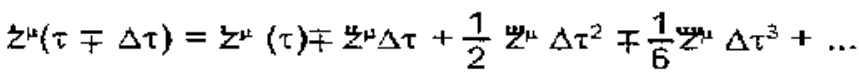

e

$$
z^{\mu}(\tau \mp \Delta \tau)=z^{\mu}(\tau) \mp z^{\mu} \Delta \tau+\frac{1}{2}{ }^{n \mu} \Delta \tau^{2} \mp \ldots
$$

a partir das quais obternos as demais expansōes, até a ordem $(\Delta \tau)^{2}$ ou $(\Delta \tau)^{3}$, onde necessário:

$$
\begin{gathered}
R_{ \pm}^{\mu}=x^{\mu}-z^{\mu}(\tau \mp \Delta \tau) \cong \pm z^{\mu} \Delta \tau+\frac{1}{2} z^{\mu} \Delta \tau^{\bar{c}} \mp \frac{1}{6} z^{\mu} \Delta \tau^{3}-\ldots,( \\
\rho_{ \pm}=-R_{ \pm}^{\mu} z_{\mu}(\tau \mp \Delta \tau)= \pm c^{2} \Delta \tau\left(1+\frac{1}{6} \frac{a^{2}}{c^{2}} \Delta \tau^{2}\right) \\
R_{\mu} z_{v} f_{ \pm}=z_{\mu} z_{v} \Delta \tau-\left(z_{\mu} z_{\nu}+\frac{1}{2} z_{\mu} z_{v}\right) \Delta \tau^{2}+ \\
\quad \pm\left(\frac{1}{2} z_{\mu} z_{v}+\frac{1}{2} z_{\mu} z_{v}+\frac{1}{6} z_{\mu} z_{v}\right) \Delta \tau^{3}
\end{gathered}
$$

$\left.\left(R_{\mu} z_{v}-R_{v} z_{\mu}\right)\right|_{2}=\frac{1}{2}\left(z_{\mu} z_{v}-z_{v} z_{\mu}\right) \Delta \tau^{2}+\frac{2}{6}\left(z_{\mu} z_{v}-z_{v} z_{F}\right) \Delta \tau^{3}$,

$\left.R_{\mu} z_{\nu}\right|_{ \pm}=\left( \pm z_{\mu} \Delta \tau-\frac{1}{2} z_{\mu} \Delta \tau^{2}\right)\left(z_{\nu} \mp z_{v} \Delta \tau\right)$

$$
= \pm z_{\mu} z_{\nu} \Delta \tau-z_{\mu} \Psi_{v} \Delta \tau^{2}-\frac{1}{2} z_{\mu} z_{v} \Delta \tau^{2},
$$

$\left.\left(R_{\mu} z_{v}-R_{v} z_{\mu}\right)\right|_{ \pm}= \pm\left(z_{\mu} z_{v}-z_{v} z_{\mu}\right) \Delta \tau-\left(z_{\mu} z_{\nu}-z_{v} z_{\mu}\right) \Delta t^{2}$,

$$
(R a)=\left(R^{\mu} z_{k}\right)=\frac{1}{2} a^{2} \Delta \tau^{2} .
$$

Substituindo estas expressôes na equaçāo dos campos (31), e tomando o limite $\Delta t \rightarrow 0$, abtemos os campos retardado

$F^{+}{ }_{\mu v}=-\frac{Q}{2 c^{4} \Delta \tau}\left(z_{\mu} z_{v}-z_{v} z_{\mu}\right)+\frac{2}{3} \frac{Q}{c^{4}}\left(z_{\mu} z_{v}-z_{v} z_{\mu}\right)$

eavançado

$F_{\mu v}^{-}=-\frac{Q}{2 c^{4} \Delta \tau}\left(z_{\mu} z_{v}-z_{v} z_{\mu}\right)-\frac{2}{3} \frac{Q}{C^{4}}\left(z_{\mu} z_{v}-z_{v} \psi_{\mu}\right)$.

Os dois campos, isoladamente, apresentam um termo divergente de ordem $(\Delta t)^{-1}$, que se cancelam no campo resultante

$$
F_{\mu \nu}=\frac{1}{2}\left(F^{+}{ }_{\mu \nu}-F_{\mu \nu}^{-}\right)=+\frac{2}{3} \frac{Q}{c^{4}}\left(z_{\mu} z_{\nu}-z_{\nu}{ }_{\mu \nu}\right) .
$$

Em modelos que não recorrem ao campo avançado, tal termo divergente é absorvido peio termo de massa da equação de movimento, num procedimento conhecido como renormalização de massa [ROHRLICH, 1965].

Se una força externa atuar sobre a carga teste de modo a mantê-la sempre próximo ao centro da esfera, de modo que $k^{\mu} \cong z^{*}$, a interação electromagnética adicional devido as cargas superficiais da casca esférisa será, para $Q=-e$ e $q=e$,

$F^{\mu}=\frac{q}{c} F^{a v} x_{v} \cong-\frac{2 e^{2}}{3 c^{5}}\left(z^{\mu} z^{v}-z^{v} z^{\mu}\right) z_{v}=\frac{2 e^{2}}{3 c^{5}}\left(a^{2} v^{\mu}-c^{2} a^{\mu}\right)$,

que é a mesma expressāo da força de LorentzDirac, a menos do sinal global. Evidenternente. no limite $v / c \rightarrow 0$, resulta na expressāo não relativística de Abrahan-Lorentz (a menos do sinal),

$$
F^{i} \cong-\frac{2}{3} \frac{e^{2}}{c^{3}} a^{i}
$$

A presença do sinal negativo nas equações (49) e (50) em reiação a equação de Lorentz-Dirac deve-se ao fato de termos considerado a casca esférica acelerada. A idéia de interação de Mach impõe uma situação diversa, devendo a casca esférica permanecer em repouso, em relação a qual a carga teste deve estar acelerada. Para se explicar a origem da força de reação radiativa como uma interação de Mach, é necessário que no referencial de repouso da carga teste apareça um campo do tipo dado peia equação (48) devido às cargas distribuídas na casca esférica. Não é uma conclusão que se possa tirar sem levar em conta um estudo mais cuidadoso do comportamento do campo eletromagnético em referenciais acelerados. 


\section{Conclusões}

Mostramos neste trabalho que forças do tipo Lorentz-Dirac, associadas ao efeito de reação radiativa de cargas aceleradas, podem aparecer em outras situações, sem nenhuma conexão aparente com fenômenos ligados à emissão de radiação eletromagnética.

No entanto, exploramos tal sistema justamente por acreditarque este resultado não seja uma coincidência puramente casual, podendo-se conjecturar que a força de reação radiativa que atua sobre uma carga q seja devido a interação da mesma com o campo eletromagnético gerado pela distribuição esfericamente simétrica das cargas complementares (carga totai $-q$ ) do Universo como um todo, numa versão do efeito Mach eletromagnético. Uma conclusão segura a este respeito depende de maiores estudos do comportamento dos campos eletromagnéticos em referenciais não inerciais, para podermos estudar o problema no referencial próprio, não inercial, da carga q, em relação ao qual o Universo como um todo pode ser visto acelerado.

GOTO, M. A Macroscopic Model for Radiation Reaction Force. Semina: Ci. Exatas/Tecnológicas, Londrina, v. 16, n. 4, p. 563-568, Dec. 1995.

ABSTRA CT: We present a simple macroscopic system, a spherical shell with a uniform charge distribution, inside of which we put a test charge. We show that the spherical shell charge distribution, when acceierated, produces a non null electromagnetic field that acts on the test charge inducing, near the spherical shell center, force such as the Lorentz-Dirac radiation reaction one.

KEY-WORDS: Radiation reaction force; Lorentz-Dirac force; Charged particles dynamic.

\section{REFERÊNCIAS BIBLIOGRÁFICAS}

BARUT, A. O. \& DOWLING, J. P. Quantum electrodynamics based on seff-fields: On the origin of themal radiation detected by an accelerating obsenver. Phys. Review A, n. 41, p. $2277-2283,1990$.

BIRRELL, N. D. \& DAVIES, P. C. W. Quantum fields in curved space. Cambridge : Cambridge Un. Press, 1982.

DIRAC, P. A. M. Classical theory of radiating electrons. Proc. Roy. Soc. A, n.167, p.148-168, 1938.

FULTON, T. \& ROMRLICH, F. Classical Radiation from a Uniformly Accelerateo Charge. Anmals of Physics, n. 9, p.499-517, 1960.

GoTo, M. Un Novo Significada Fisico para os Campos Avançados, Revista Brasileira de Ensino de Fisica, v. 17, n. 4, p. 270-278, 1995.

INFELD, L. \& WALLACE, P. R. The equations of motion in electrodynamics. Phys. Rev, n.57, p.797-806, 1940.

JACKSON, J. D. Classical Electrodynamics. New York: John Wiley \& Sons, 1962.

LANDAU, L. \& LIFSHITZ, E. L. Tearia do Campo. MoscoU: MIR, 1980.
MARION, J. B. Classical Electromagnetic Radiation. London : Academic Press, 1965.

MOLLER, C. The Theory of Relativity (second edition). New York : Oxford University Press, 1972.

ROHRl.ICH, F. The Equations of Motion of Classical Charges. Ann. of Physics, n. 13, p. 93-109, 1961.

ROHRLICH, F. The Principle of Equivalence, Amnats of Physics, v. 22, p. 189-191, 1963.

ROHRLICH, F. Classical Charged Particles. New York: Addison-Wesley, 1965.

TEITELBOIN, C.; VILLARROEL, D. \& WEERT, Ch. G. Van Classical Electrodynamics of Retarded Field's and Point Panticles. Rev. Nuovo Cimento, v. 3, n. 9, p. 1-64. 1980.

UNRUH, W. G. Notes on black-hole evaporation. Phys. Review D, ח. 14, p. 870-892, 1976.

UNRUH, W. G. \& WALD, R. M. What happens when an accelerating observer detects a Rindier particle. Phys. Review $D$, n. 29, p. 1047-1056, 1984.

WEINBERG, S. Gravitation and Cosmolagy. New York: John Wiley \& Sons, 1972. 